\title{
EFFECT OF STABILITY INDICES ON ROBUSTNESS AND SYSTEM RESPONSE IN COEFFICIENT DIAGRAM METHOD
}

\author{
Surekha Bhusnur ${ }^{1}$ \\ ${ }^{1}$ Professor, Department of Electrical and Electronics Engineering, BIT, Durg, C.G., India
}

\begin{abstract}
In Control systems, designing a robust controller such that a desired system response is obtained despite plant parameter variations is ubiquitous problem. In this context, Coefficient diagram method is an effective method and one of the recent design methods based on the polynomial approach introduced by Shunji Manabe. In CDM, stability indices, stability limits and time constant are the main design parameters. The stability indices and stability limits are indicative of stability and equivalent time constant is indicative of speed of system response. A semi-log diagram known as coefficient diagram is the design tool using which one can analyse the important features of a design such as stability, speed of response and robustness, all in one diag ram. The right choice of the stability indices is of paramount importance in the controller design. This paper deals with the effect of variation in the stability indices upon the system response and robustness. A type 2 fourth order plant has been considered as an example to analyse the effects of stability indices. The stability indices are varied one by one relative to the standard Manabe form and in each case response is observed. The transient response of the system is sensitive to lower order indices. Also, robustness in the design is analysed by coefficient diagrams of the perturbed plant.
\end{abstract}

Keywords: Coefficient Diagram Method (CDM), robustness; Stability Indices, Coefficient Diagram

\section{INTRODUCTION}

In CDM [1, 2], firstly, the type and degree of the controller polynomial and the closed loop transfer function are partially specified and later other parameters are obtained by design. The key features of the method are: adaptation of the polynomial representation for both the plant and the controller, this avoids pole-zero cancellations; use of two degree of freedom control structure; almost no overshoot in the step response of the closed loop system; the determination of the settling time at the start, good robustness with respect to parameter changes [3, 5, 7]. A brief survey on CDM is presented in [9]. Preliminaries of CDM have been briefly introduced in many contributions based on CDM [8, 10, 11, 12, 15, 16, 17]. The comparative study of other control design methods with CDM is found in [11].

In CDM the design parameters are Stability index $\gamma_{i}$ Stability limit $\gamma_{i}{ }^{*}$ and Equivalent time constant $\tau$. The stability indices and stability limits are indicative of system stability; and the equivalent time constant is indicative of speed of response. In CDM design, the given design specifications are rewritten in terms of $\gamma_{i}$ and $\tau$. These parameters are related to controller parameters algebraically and specify the target characteristic equation that gives desired performance. A special diagram known as Coefficient Diagram is used as a design tool using which the coefficients of the characteristic polynomial can be re-tuned to get the required system performance. CDM is one such algebraic method which gives the most proper results with the easiest procedure.
Rest of the paper is organized as follows:

In section 2, the control structure used in CDM is described in brief. It includes a pre-filter to adjust steady state gain [1, $3,6,7]$. In section 3 , the stability and instability conditions used in CDM are discussed and relevant mathematical relations are listed in terms of stability indices. These conditions have been presented in terms of design parameters used in CDM [3]. In section 4, the standard Manabe form of CDM design and its features are briefed [3, 5]. Coefficient diagram, the design tool of CDM [7] is explained with an example in Section 5. Also, the design steps involved in controller design are enlisted in Section 5. In section 6 the effect of variation in stability indices on the system response is discussed considering a fourth order plant transfer function taken from existing literature [4]. Also, simulation results are discussed focusing the role of stability indices upon the transient response of the closed loop system and robustness. Section 7 is the concluding part.

\section{CONTROL STRUCTURE}

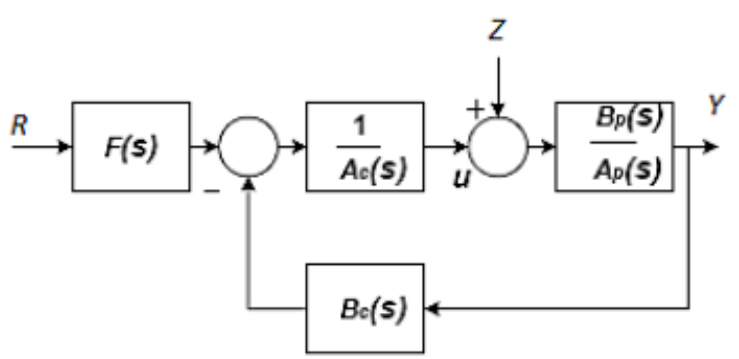

Fig-1: Block diagram of CDM control 
The standard block diagram for CDM $[2,7,8,12]$ is shown in Fig. 1. Here, $R(s)$ is the reference input, $Y(s)$ is the output, $u$ is the control and $Z(s)$ is the external disturbance signal. The effect of external disturbance has not been considered in this paper as the objective is to analyze the utility of stability indices. $B_{p}(s)$ and $A_{p}(s)$ are the numerator and denominator polynomials of the transfer function of the plant respectively. $F(s)$ and $B_{c}(s)$ are the reference numerator and the feedback numerator polynomials while $A_{c}(s)$ is the forward denominator polynomial of the controller transfer function. $B_{c}(s)$ and $A_{c}(s)$ are designed to meet the desired transient response and the pre-filter $F(s)$ is used to provide the steady state gain.

The output of this closed loop system is

$$
Y(s)=\frac{B_{p}(s) F(s)}{A_{c l}(s)} R(s)+\frac{A_{c}(s) B_{p}(s)}{A_{c l}(s)} Z(s)
$$

Where $A_{c l}(s)$ is the characteristic polynomial and is given by

$$
\begin{gathered}
A_{c l}(s)=A_{p}(s) A_{c}(s)+B_{p}(s) B_{c}(s) \\
=a_{n} s^{n}+\ldots \ldots \ldots . . .+a_{1} s+a_{0} \\
=\sum_{i=0}^{n} a_{i} s^{i}
\end{gathered}
$$

The design parameters of CDM the equivalent time constant $\tau$, the stability index $\gamma_{i}$, and stability limit $\gamma_{i}^{*}$ are defined as

$$
\begin{aligned}
& \tau=\frac{a_{1}}{a_{0}} \\
& \gamma_{i}=\frac{a_{i}^{2}}{a_{i+1} a_{i-1}}, i=1,2, \ldots \ldots \ldots . .(n-1) \\
& \quad \gamma_{i}^{*}=\frac{1}{\gamma_{i+1}}+\frac{1}{\gamma_{i-1}}, i=1,2, \ldots \ldots . .(n-1), \gamma_{n}, \gamma_{0}=\infty
\end{aligned}
$$

The equivalent time constant specifies the speed of time response, the stability indices and limits specify stability, nature of time response; and the variation of stability indices due to plant perturbations indicates the robustness $[2,3]$.

\section{STABILITY CONDITIONS}

The stability conditions suitable to $\mathrm{CDM}$ are based on Lipatov conditions and are stated in Theorem 4 in [2, 3] as follows: "The system is stable if all the partial fourth order polynomials of the characteristic equation are stable with a margin of 1.12. The system is unstable if some partial third order polynomial is unstable".
- Thus the sufficient condition for stability is given as

$$
\gamma_{i}>1.12 \gamma_{i}^{*}, i=1,2, \ldots \ldots .(n-2)
$$

- The sufficient condition for instability is given by

$$
\left(\gamma_{i+1} \gamma_{i} \leq 1,\right) \text { forsome } i=1,2, \ldots \ldots .(n-2)
$$

\section{STANDARD FORM}

Shunji Manabe suggested values of stability indices such that a response with no overshoot is obtained. This new form is known as "Standard Manabe Form" of CDM and is given by [1]

$$
\gamma_{1}=2.5, \gamma_{n-1}=\ldots \ldots \ldots . \gamma_{2}=2
$$

The advantages of standard form are enlisted below [7]

- for system type 1 , overshoot is almost zero

- the Manabe form has shortest settling time among the systems with the same equivalent time constant $\tau$ and the value is about $2.5 \tau \sim 3 \tau$

- The step responses show almost equal wave forms irrespective of the order of the characteristic polynomials

- the lower order poles are aligned almost on a vertical line

- The values of stability indices in Manabe Form are easy to remember

\section{COEFFICIENT DIAGRAM AND \\ CONTROLLER DESIGN}

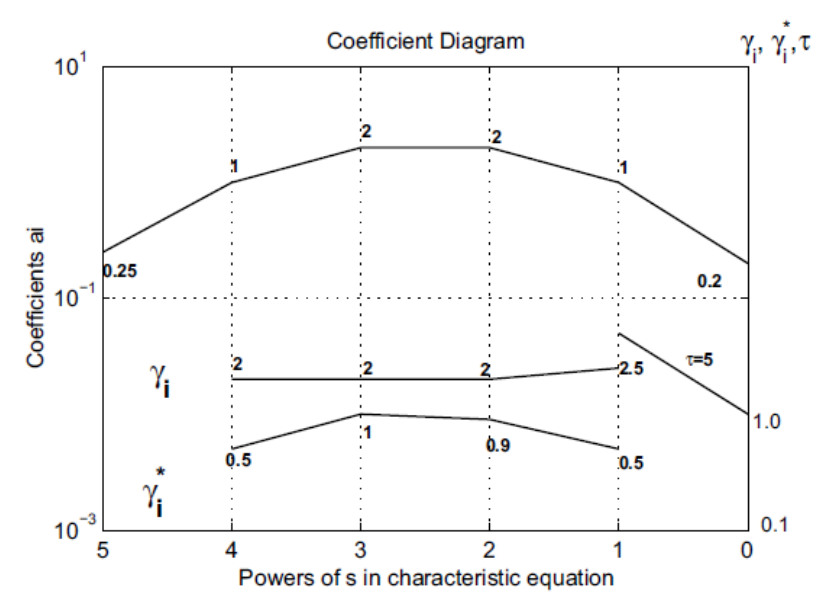

Fig-2: Coefficient diagram

The Coefficient Diagram (CD) is a semi-log diagram of the coefficients $a_{i}$ of the polynomial in logarithmic scale on left hand side vertical axis and the corresponding powers of $s$ are placed in decreasing order in linear scale on horizontal axis, also the stability index, stability limit and the equivalent time constant are read on right hand side vertical axis. The equivalent time constant is expressed by a line connecting 1 to $\tau$. A Coefficient Diagram for the characteristic polynomial given by 


$$
A_{c l}(s)=0.25 s^{5}+. s^{4}+2 s^{3}+2 s^{2}+s+0.2
$$

is shown in Fig. 2. Greater the curvature of the coefficient curve the more the system stability. The smaller the value of $\tau$, the steeper is the left end part of the coefficient curve and the faster is the system response. The change in the shape of coefficient curve due to changes in parameters is an indicative of robustness and sensitivity to variation in parameters.

The controller design steps $[2,7]$ are summarized as follows:

- Express the plant transfer function as ratio of numerator and denominator polynomials.

- Translate the given performance specifications into design specifications for CDM.

- Assume a suitable controller configuration and express in the polynomial form.

- Solve for the unknown parameters using Diophantine equation.

- Draw Coefficient Diagram, visualize and make adjustments in the relevant coefficients, if necessary to satisfy the performance specifications.

\section{EFFECT OF VARIATION IN STABILITY INDICES}

\section{Simulation Example}

A fourth order plant transfer function [4] has been considered in this section to analyse the effect of variation in stability indices $\gamma_{i}$.

The real plant is represented as

$$
\begin{gathered}
P(s)=\frac{B_{p}(s)}{A_{p}(s)}=\frac{K_{m}}{s^{2}(s+a)(s+b)}, \\
K_{m} \in[8,12], a \in[0.5,1.5], b \in[2,4]
\end{gathered}
$$

A third order controller is designed using CDM as described in Section 5. The controller transfer function is assumed as

$$
\frac{B_{c}(s)}{A_{c}(s)}=\frac{k_{3} s^{3}+k_{2} s^{2}+k_{1} s+k_{0}}{l_{3} s^{3}+l_{2} s^{2}+l_{1} s+l_{0}}
$$

From (2) and (10) we see that the characteristic polynomial is of order 7. Using (6) and (8) the stability indices are assumed to have six sets of values as given in Table 1 to observe the effect in system response.

Table 1. Assumed values of stability indices

\begin{tabular}{|l|l|l|l|l|l|l|}
\hline $\begin{array}{l}\text { Se } \\
\mathbf{t}\end{array}$ & $\gamma_{1}$ & $\gamma_{2}$ & $\gamma_{2}$ & $\gamma_{4}$ & $\gamma_{5}$ & $\gamma_{6}$ \\
\hline 1 & $\begin{array}{l}2.5,4,6, \\
8\end{array}$ & 2 & 2 & 2 & 2 & 2 \\
\hline 2 & 2.5 & $\begin{array}{l}2,4,6, \\
8\end{array}$ & 2 & 2 & 2 & 2 \\
\hline 3 & 2.5 & 2 & $\begin{array}{l}2,4,6, \\
8\end{array}$ & 2 & 2 & 2 \\
\hline
\end{tabular}

\begin{tabular}{|l|l|l|l|l|l|l|}
\hline 4 & 2.5 & 2 & 2 & $\begin{array}{l}2,4,6, \\
8\end{array}$ & 2 & 2 \\
\hline 5 & 2.5 & 2 & 2 & 2 & $\begin{array}{l}2,4,6, \\
8\end{array}$ & 2 \\
\hline 6 & 2.5 & 2 & 2 & 2 & 2 & $\begin{array}{l}2,4,6, \\
8\end{array}$ \\
\hline
\end{tabular}

A settling time specification of $t_{s} \leq 5 \mathrm{sec}$ has been assumed in the controller design. For every set, four controllers are designed and system responses are plotted using MATLAB and SIMULINK environment, the controller parameters are enlisted in Table 2. In each case the responses obtained are shown in Fig. 3 to Fig. 8. It is observed that $\gamma_{1}$ and $\gamma_{2}$ have greater influence on the system response, whereas increase in higher order indices increases robustness. Also, the controller parameters deviate less for higher order indices as depicted in Table 2.

Further to observe robustness coefficient diagrams are plotted for the eight plants of the family and are shown in Fig. 9. There is less deviation in the wave shape of the coefficient curves for the perturbed family of plants and indicates robustness. Also, stability is ensured by stability conditions given in Section 3 and the ratio of stability

\begin{tabular}{|c|c|c|c|c|c|c|c|c|}
\hline & 13 & 12 & 11 & 10 & $k 3$ & $k 2$ & $k 1$ & $k 0$ \\
\hline & $\begin{array}{l}1.60 \\
00 \mathrm{e}-\end{array}$ & $\begin{array}{l}5.76 \\
00 \mathrm{e}\end{array}$ & $\begin{array}{l}0.01 \\
04\end{array}$ & $\begin{array}{l}0.0 \\
845\end{array}$ & $\begin{array}{l}0.0 \\
271\end{array}$ & $\begin{array}{l}0.1 \\
347\end{array}$ & $\begin{array}{l}0.2 \\
000\end{array}$ & $\begin{array}{l}0.1 \\
000\end{array}$ \\
\hline \multirow{5}{*}{ 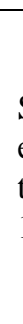 } & 9.53 & 5.72 & 0.00 & 0.0 & 0.0 & 0.0 & 0.2 & 0.1 \\
\hline & $67 \mathrm{e}-$ & $20 \mathrm{e}-$ & 17 & 242 & 148 & 927 & 000 & 000 \\
\hline & 8.37 & 7.70 & 0.00 & $\overline{0.0}$ & 0.0 & 0.0 & 0.2 & 0.1 \\
\hline & $24 \mathrm{e}-$ & $27 \mathrm{e}-$ & 04 & 078 & 079 & 643 & 000 & 000 \\
\hline & 1.49 & 1.84 & 1.14 & 0.0 & 0.0 & 0.0 & 0.2 & 0.1 \\
\hline & $01 \mathrm{e}-$ & $77 \mathrm{e}-$ & $63 \mathrm{e}-$ & 034 & 048 & 490 & 000 & 000 \\
\hline & 1.60 & 0.00 & 0.01 & 0.0 & 0.0 & 0.1 & 0.2 & 0.1 \\
\hline \multirow{6}{*}{$\begin{array}{l}\mathrm{S} \\
\mathrm{e} \\
\mathrm{t} \\
2\end{array}$} & $00 \mathrm{e}-$ & 06 & 04 & 845 & 271 & 34 & 000 & 000 \\
\hline & 5.00 & 3.80 & 0.00 & 0.0 & 0.0 & 0.1 & 0.2 & 0.1 \\
\hline & $00 \mathrm{e}-$ & $00 \mathrm{e}-$ & 14 & 261 & 211 & 522 & 000 & 000 \\
\hline & 6.58 & 7.63 & 4.43 & 0.0 & 0.0 & 0.1 & 0.2 & 0.1 \\
\hline & $44 \mathrm{e}-$ & 79e- & $33 \mathrm{e}-$ & 124 & 162 & 563 & 000 & 000 \\
\hline & 1.56 & 2.43 & 1.90 & 0.0 & 0.0 & 0.1 & 0.2 & 0.1 \\
\hline & $25 \mathrm{e}-$ & $75 \mathrm{e}-$ & $20 \mathrm{e}-$ & 072 & 131 & 578 & 000 & 000 \\
\hline \multirow{7}{*}{$\begin{array}{l}\mathrm{S} \\
\mathrm{e} \\
\mathrm{t} \\
3\end{array}$} & 1.60 & 0.00 & 0.01 & 0.0 & 0.0 & 0.1 & 0.2 & 0.1 \\
\hline & $\frac{00 \mathrm{e}-}{100}$ & $\frac{06}{0.00}$ & $\frac{04}{000}$ & $\frac{845}{00}$ & $\frac{271}{00}$ & $\frac{347}{01}$ & $\frac{000}{02}$ & $\frac{000}{01}$ \\
\hline & $00 \mathrm{e}-$ & 01 & 29 & 522 & 423 & 443 & 000 & 000 \\
\hline & 1.97 & 2.29 & 0.00 & 0.0 & 0.0 & 0.1 & 0.2 & $\begin{array}{l}0.1 \\
\end{array}$ \\
\hline & $53 \mathrm{e}-$ & $14 \mathrm{e}-$ & 13 & 373 & 487 & 488 & 000 & 000 \\
\hline & 6.25 & 9.75 & 7.60 & 0.0 & 0.0 & 0.1 & 0.2 & 0.1 \\
\hline & 00e- & 00e- & 81e- & 289 & 522 & 513 & 000 & 000 \\
\hline \multirow{6}{*}{$\begin{array}{l}\mathrm{S} \\
\mathrm{e} \\
\mathrm{t} \\
4\end{array}$} & 1.60 & 5.76 & 0.01 & 0.0 & 0.0 & 0.1 & 0.2 & 0.1 \\
\hline & $00 \mathrm{e}-$ & 00e- & 04 & 845 & 271 & 347 & 000 & 000 \\
\hline & 2.00 & 0.00 & 0.00 & 0.1 & 0.0 & 0.1 & 0.2 & 0.1 \\
\hline & O0e- & 02 & 58 & 044 & 205 & 287 & 000 & 000 \\
\hline & 5.92 & 0.00 & 0.00 & 0.1 & 0.0 & 0.1 & 0.2 & 0.1 \\
\hline & $59 \mathrm{e}-$ & 01 & 40 & 118 & 181 & 264 & 000 & 000 \\
\hline
\end{tabular}
indices to stability limits is enlisted for all the eight plants in Table 3.

Table 2. Controller parameters 


\begin{tabular}{|c|c|c|c|c|c|c|c|c|}
\hline & $\begin{array}{l}2.50 \\
00 \mathrm{e}-\end{array}$ & $\begin{array}{l}3.90 \\
00 \mathrm{e}-\end{array}$ & $\begin{array}{l}0.00 \\
30\end{array}$ & $\begin{array}{l}0.1 \\
157\end{array}$ & $\begin{array}{l}0.0 \\
168\end{array}$ & $\begin{array}{l}0.1 \\
253\end{array}$ & $\begin{array}{l}0.2 \\
000\end{array}$ & $\begin{array}{l}0.1 \\
000\end{array}$ \\
\hline \multirow{8}{*}{$\begin{array}{l}\mathrm{S} \\
\mathrm{e} \\
\mathrm{t} \\
5\end{array}$} & 1.60 & 0.00 & 0.01 & 0.0 & 0.0 & 0.1 & 0.2 & 0.1 \\
\hline & $00 \mathrm{e}-$ & 06 & 04 & 845 & 271 & 347 & 000 & 000 \\
\hline & 4.00 & 0.00 & 0.01 & 0.0 & 0.0 & 0.1 & 0.2 & 0.1 \\
\hline & $00 \mathrm{e}-$ & 03 & 16 & 808 & 282 & 358 & 000 & 000 \\
\hline & 1.77 & 0.00 & 0.01 & 0.0 & 0.0 & 0.1 & 0.2 & 0.1 \\
\hline & $78 \mathrm{e}-$ & 02 & 20 & 795 & 286 & 361 & 000 & 000 \\
\hline & 1.00 & 0.00 & 0.01 & 0.0 & 0.0 & 0.1 & 0.2 & 0.1 \\
\hline & $00 \mathrm{e}-$ & 02 & 22 & 788 & 288 & 363 & 000 & 000 \\
\hline \multirow{8}{*}{$\begin{array}{l}\mathrm{S} \\
\mathrm{e} \\
\mathrm{t} \\
6\end{array}$} & 1.60 & 0.00 & 0.01 & 0.0 & 0.0 & 0.1 & 0.2 & 0.1 \\
\hline & $00 \mathrm{e}-$ & 06 & 04 & 845 & 271 & 347 & 000 & 000 \\
\hline & 8.00 & 0.00 & 0.01 & 0.0 & 0.0 & 0.1 & 0.2 & 0.1 \\
\hline & $00 \mathrm{e}-$ & 06 & 03 & 848 & 270 & 346 & 000 & 000 \\
\hline & 5.33 & 0.00 & 0.01 & 0.0 & 0.0 & 0.1 & 0.2 & 0.1 \\
\hline & $33 \mathrm{e}-$ & 06 & 03 & 849 & 269 & 345 & 000 & 000 \\
\hline & 4.00 & 0.00 & 0.01 & 0.0 & 0.0 & 0.1 & 0.2 & 0.1 \\
\hline & $00 \mathrm{e}-$ & 06 & 03 & 850 & 269 & 345 & 000 & 000 \\
\hline
\end{tabular}

In Fig. 3, nominal plant is considered and the results show that the transient response deviates from that of Standard form and settling time increases, transient response is affected by the lower order indices, $\gamma_{1}$ and $\gamma_{2}$. Also, it is seen that increase in higher order indices $\gamma_{3}, \gamma_{4}, \gamma_{5}$ and $\gamma_{6}$ least affects the transient response and the responses are similar to that of the response corresponding to the standard Manabe form.

In Fig. 9, perturbed plant, with the parameters set to extreme values of the interval box given in (10) is considered and coefficient diagrams for all the eight plants of the family are plotted, the results show that the standard Manabe form based controllers give robust performance despite plant parameter variations.

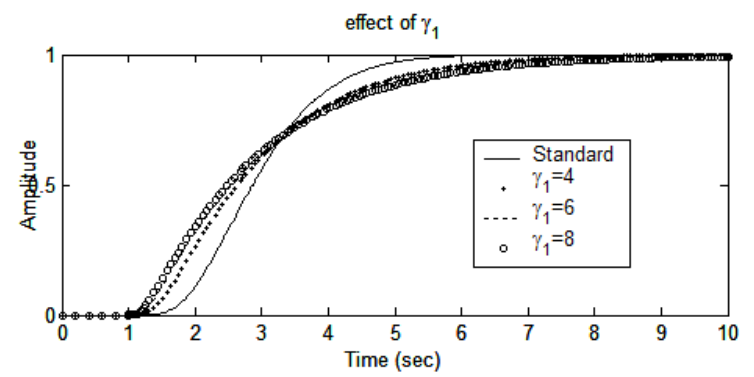

Fig-3: Effect of $\gamma_{1}$

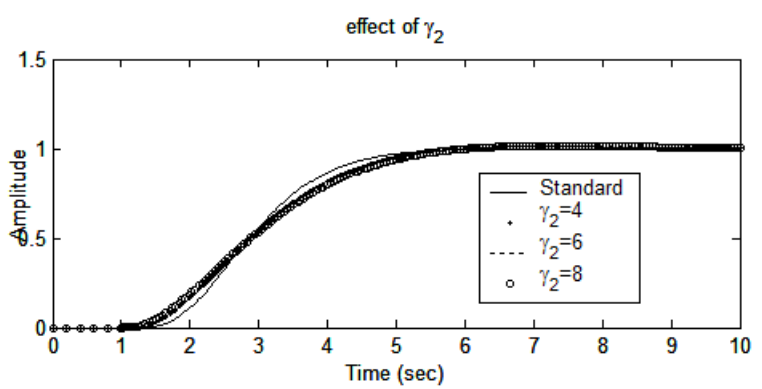

Fig-4: Effect of $\gamma_{2}$

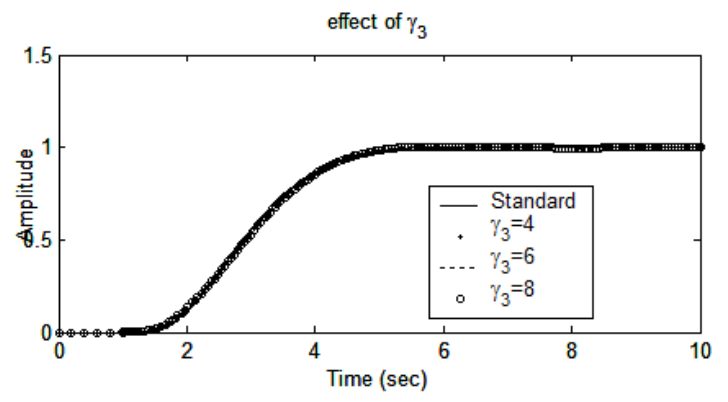

Fig-5: Effect of $\gamma_{3}$

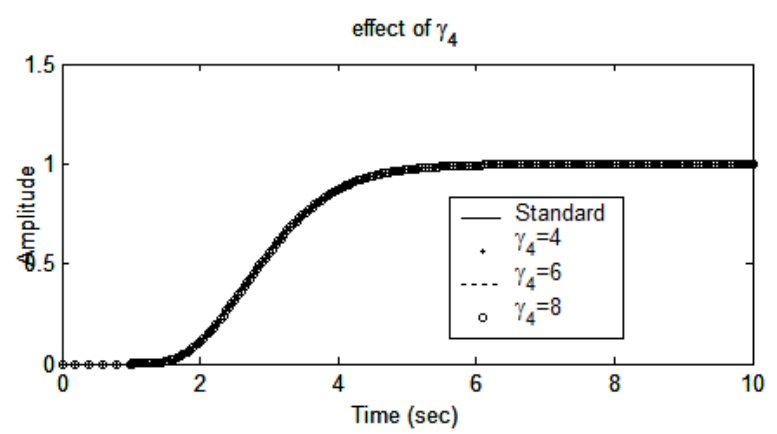

Fig-6: Effect of $\gamma_{4}$

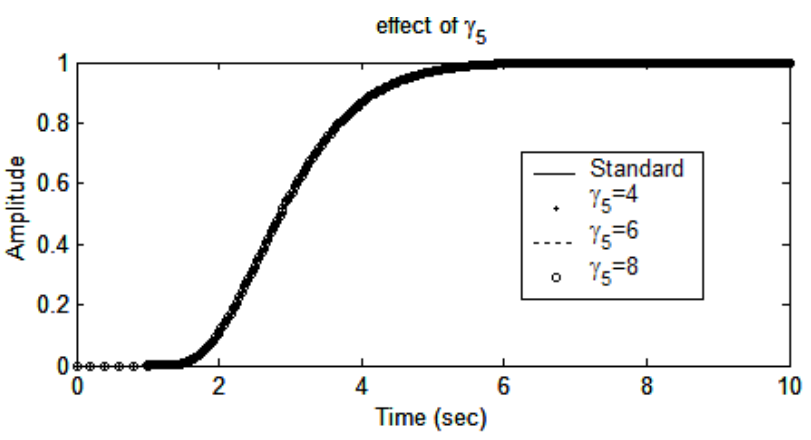

Fig-7: Effect of $\gamma_{5}$

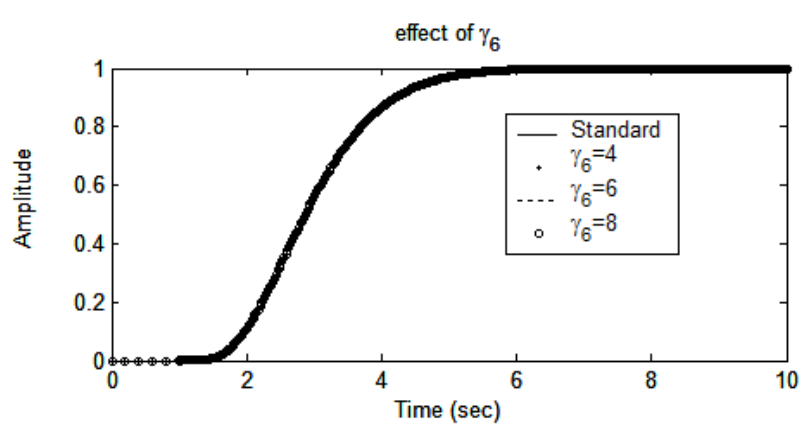

Fig-8: Effect of $\gamma_{6}$ 


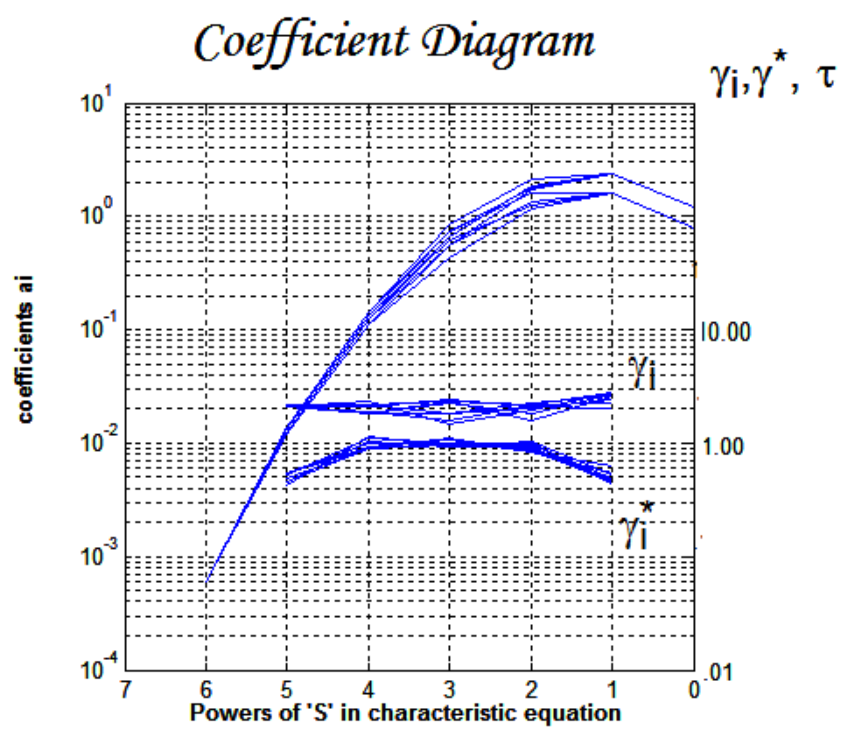

Fig-9 Coefficient Diagrams

\section{CONCLUSION}

In this paper, the behavior of a simple fourth order plant subjected to variation in stability indices in CDM based design has been described. Emphasis has been given to have an insight to the way, transient response and robustness features are affected. An ideal linear nominal model of the plant and a CDM based controller has been employed to generate output corresponding to standard form. The effects of variation in stability indices and robust stability considering the perturbed plant have been analyzed. It is observed that the lower order indices influence the transient behavior the most and the higher order indices can be relaxed from the standard form values to improve robustness. As the role of stability indices has been focused disturbance rejection and the other properties have been ignored.

Table 3: Quantitative Robust stability analysis

\begin{tabular}{|l|l|l|l|l|l|}
\hline $\begin{array}{l}\text { Sl. } \\
\text { No. }\end{array}$ & \multicolumn{5}{|l|}{$\gamma_{\mathrm{i}} / \gamma_{\mathrm{i}}{ }^{*}>1.12$} \\
\hline 1 & 5.0256 & 2.0709 & 1.5804 & 1.8604 & 5.3009 \\
\hline 2 & 4.6822 & 2.4210 & 2.1065 & 1.9118 & 4.0353 \\
\hline 3 & 4.7011 & 2.1640 & 1.9208 & 2.1031 & 4.8960 \\
\hline 4 & 4.4596 & 2.3526 & 2.5123 & 2.3151 & 4.2600 \\
\hline 5 & 4.0294 & 1.7202 & 1.6134 & 2.2350 & 6.2207 \\
\hline 6 & 3.9834 & 2.0239 & 2.0479 & 2.2290 & 4.9168 \\
\hline 7 & 3.9197 & 1.8274 & 1.8741 & 2.4046 & 5.7346 \\
\hline 8 & 3.8924 & 2.0333 & 2.3388 & 2.5421 & 4.9825 \\
\hline
\end{tabular}

\section{REFERENCES}

[1] S. Manabe,(1997) "A solution of the acc benchmark problem by coefficient diagram method", in Proceedings of 2nd Asian Control Conference, July 22-25, Seoul, II-135-138.
[2] S. Manabe,( 1998) "Coefficient diagram method", Proc. 14th IFAC Symposium on Automatic Control in Aerospace, Seoul, Korea, August.

[3] S. Manabe, (1999)"Sufficient condition for stability and instability by Lipatov and its application to the coefficient diagram method", in Proceedings of 9th Workshop on Astrodynamics and Flight Mechanics, ISAS, Sagamihara, pp.440-449.

[4] J.J.D. Azzo and C. H. Houpis,( 1988) “ Linear Control System Analysis and Design:Conventional and Modern", McGraw-Hill, third edition.

[5] S. Manabe and Y.-C. Kim,( 2000) "Recent development in coefficient diagram method", in Proceedings of 3rd Asian Control Conference, Shanghail, July 4-7.

[6] A. Uqar and S. E. Hamamci,( 2000) “A controller based on coefficient diagram method for the robotic manipulators", in Proceedings of the 7th IEEE Conference on Electronics, Ciruits and Systems, ICECS, volume 2.

[7] Y. C. Kim and S. Manabe,( 2001) “ Introduction to coefficient diagram method", in Proceedings of SSSC'01, Prague.

[8] S. E. Hamamci and M. Koksal,( 2001) "Robust control of a dc motor by coefficient diagram method", in Proceedings of MED'01 The 9th Mediterranean Conference on Control and Automation, Dubrovnik, Crotia, June 27-29.

[9] S. Manabe, (2002) “ Brief tutorial and survey of coefficient diagram method", in Proceedings of The $4^{\text {th }}$ Asian Control Conference, Singapore.

[10] S. Manabe,( 2003) "Importance of coefficient diagram in polynomial method", in Proceedings of $42^{\text {nd }}$ IEEE Conference on Decision and Control, Maui, HI, pp.3489-3494.

[11] H. Kim, (2004) "The study of control design method", in Proceedings of 8th Russian-Korean International Symposium on Science and Technology, KORUS, volume 1, pages 55-58.

[12] S. E. Hamamci,(2005) "A robust polynomial-based control for stable processes with time delay", Electrical Engineering, 87:163-172.

[13] D. R. Choudhary, (2005) Modern control engineering. Prentice-Hall of India Private Limited, New Delhi., first edition.

[14] M. Gopal, Control systems,(2006) (principles and design)Tata McGraw-Hill Publishing Company Private Limited, New Delhi., second edition.

[15] P. Pattanavij,(2006) "Simplified design of pi controller based on cdm", in Proceedings of SICEICASE International Joint Conference.

[16] S. Bhusnur and S. Ray, (2010) "A novel robust control strategy for interval plants using the two loop mfc and cdm", in Proceedings of 4th International Workshop on Reliable Engineering Computing (REC 2010), Singapore, pages 665-683.

[17] S. Bhusnur and S. Ray.,(2011) "Robust control of integrating systems using cdm-based two-loop control structure", Int. J. Reliability and Safety, special issue on Robust Design, 5(3/4):250-269. 


\section{BIOGRAPHY}

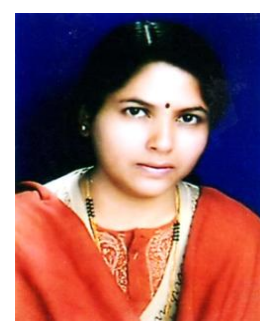

Surekha Bhusnur is a Professor in the

Department of Electrical and Electronics Engineering at Bhilai Institute of Technology, Durg, Chhattisgarh Swami Vivekanand Technical University, India. Her research interests include robust control and instrumentation. 